\title{
Modified Perona-Malik Equation and Computer Simulation for Image Denoising
}

\author{
Zheng Jian Feng ${ }^{*}, 1$, Huang Chengwei ${ }^{2}$ and Zhang $\mathrm{Ji}^{1}$ \\ ${ }^{I}$ Changzhou University, Chang Zhou, 21300, China \\ ${ }^{2}$ Soochow University, Suzhou, 21500, China
}

\begin{abstract}
The edges and textures of a digital image may be destroyed by traditional denoising methods, which is a difficult problem in image denoising. In this paper, anisotropic diffusion algorithm based on Partial differential equation is studied. First, image denoising algorithms based on Perona-Malik model are studied. Second, a modified Perona-Malik model is proposed. In the proposed model, the gradient statistic and edge thresholds are embedded into the Perona-Malik equation. Finally, the effects of this model and some other models are compared and analyzed. The experimental results show that the proposed modified Perona-Malik model outperforms the original Perona-Malik model in removing Gaussian noise, and the edges and textures of the image are well preserved.
\end{abstract}

Keywords: Computer simulation, Image denoising, Partial differential equation, Perona-Malik model.

\section{INTRODUCTION}

Due to the typical problems in image processing, image smoothing has been widely used in image displaying, transmission, analyzing, animation production, media composition and so on [1-4]. The main purpose of image smoothing is to eliminate the noise in the corrupted images. In the process of imaging and transmission, the images are often polluted by a lot of noise, which not only influences the vision effect heavily, but also causes some difficulties in image analysis and understanding. Therefore, image smoothing plays an important role in image preprocessing.

Image denoising algorithm has many important applications in Remote Sensing (RS) image processing. Remote sensing is the acquisition of information regarding an object or phenomenon without making physical contact with the object. Remote sensing images are affected by different types of noises like Gaussian noise, speckle noise and impulse noise. Therefore, remote-sensing images are usually of low quality and can be improved by image denoising algorithms.

There are many methods for image smoothing, such as Gaussian smoothing, wavelet thresholding filtering, fractal denoising, etc. The Gaussian filter is widely used in image smoothing but it destroys the textures and edges. The anisotropic diffusion model is one of the denoising models which smoothens the images selectively, while maintaining the edge remains.

Convoluting images with different scaled Gaussian filter are equivalent to transforming images with heat conduction

*Address correspondence to this author at the Changzhou University, Chang Zhou, 21300, China; Tel: 0086-519-86330284; Fax: 0086-519-88018395; E-mail: czzhengjianfeng@163.com equations but the latter demonstrates the processing of isotropic diffusion which cannot tune the smoothing velocity according to the context of the images. Based on the above analysis, Perona and Malik proposed nonlinear partial differential equation for image smoothing [5], maintaining the model image edges on smoothening the noise.

To determine diffusion velocity with image gradient, two aspects are needed to be considered; noise removal and feature preserving. As a typical example of this kind of method, Perona-Malik (PM) model has been widely used in edge detection, image enhancement, image segmentation, object recognition and so on. But with the diffusion factors proposed by Perona-Malik, diffusion velocity cannot be decreased immediately when obvious features are presented, which makes these features get eliminated unintentionally; on the other hand, velocity is slow when facing unobvious features.

Based on partial differential equations, the noise in the remote sensing image should be removed, and at the same time, the textures of the image should be well preserved. Perona-Malik model is known for its good performance in preserving image details. Hence, it is suitable for remote sensing image processing and other real world image processing.

\section{BRIEF INTRODUCTION TO PERONA-MALIK MODEL}

Let the image be defined in open square area, PeronaMalik model is [5]:

$$
\begin{aligned}
& I_{t}=\operatorname{div}(g(|| \nabla I||) \bullet \nabla I) \\
& I(x, y, 0)=I_{0}
\end{aligned}
$$


where, $\mathrm{I}$ is the function of space variables $\mathrm{x}, \mathrm{y}$ and time variable $t$, which means the image intensity of $(x, y)$ in time $\mathrm{t}$; div is the divergence operator of space variables $\mathrm{x}, \mathrm{y}$; $\mathrm{I} 0$ means original image. $\mathrm{g}(\mathrm{s})$ is a decreasing function which satisfies the following two conditions:

$$
g(0)=1 ; \lim _{s \rightarrow \infty} g(s)=0
$$

In order to solve Eq.(1), it must be discretized with different scheme and solved iteratively [6]. The discretize formulation of Perona-Malik model is

$$
\begin{aligned}
I_{i, j}^{t+1}=I_{i, j}^{t} & +\lambda\left[g_{N} \bullet \nabla_{N} I+g_{S} \bullet \nabla_{S} I\right. \\
& \left.+g_{E} \bullet \nabla_{E} I+g_{W} \bullet \nabla_{W} I\right]_{i, j}^{t}
\end{aligned}
$$

where, N, S, E and $\mathrm{W}$ are the abbreviations for North, South, East, West, respectively; $\lambda$ is the parameter to maintain the numerical stability of algorithm, which satisfies $0 \leq \lambda \leq 1 / 4 ; \nabla$ is the difference quotient instead of the gradient operator, i.e. [7]:

$$
\begin{aligned}
& \nabla_{N} I_{i, j}=I_{i, j+1}-I_{i, j} \\
& \nabla_{S} I_{i, j}=I_{i, j-1}-I_{i, j} \\
& \nabla_{E} I_{i, j}=I_{i+1, j 1}-I_{i, j} \\
& \nabla_{W} I_{i, j}=I_{i-1, j}-I_{i, j}
\end{aligned}
$$

And $g_{N_{i, j}}^{t}=g\left(\left|\nabla_{N}^{t} I_{i, j}\right|\right), g_{S_{i, j}}^{t}, g_{E_{i, j}}^{t}, g_{W_{i, j}}^{t}$ are defined similar to in Eq. (3).

P-M model controls the diffusion velocity with gfunction adaptively, i.e., increases the smoothing force in the interior of image area, and decreases it, thereby avoiding it on the image edges. In P-M model, the edges are often recognized by gradient differential operator. From the mathematical point of analysis, the P-M model is an improved heat conduction partial differential equation in essence.

The smoothing effect of P-M model is not good. When assuming that there is an isolated noise pixel, and comparing this pixel with each pixel in its neighbor intensity disparities become large, and the gradients are large inevitably. At this time, the diffusion velocity is slow, which is not beneficial for eliminating the noisy pixel. On the edge of the image, although the gradient is large, it still is influenced by the pixels in its neighbor, and the edges are blurred or destroyed after several iterations even when the influence is small.

\subsection{Selection of Heat Diffusion Coefficient}

There are two candidates for heat diffusion coefficient $\mathrm{g}$ function in P-M model, as shown in $\operatorname{Eqs}(5)$ and (6):

$$
g(s)=\exp \left[-\left(\frac{s}{k}\right)^{2}\right]
$$

where $\mathrm{k}$ is the estimate factor of noise.

Perona and Malik considered that independent variable gfunction is the best estimation for the intensity of edges of images. The estimated function is not only used to detect the edges but also has significance in statistics which can be normalized as follows [8].

$$
g(s)=\frac{1}{1+\left(\frac{s}{k}\right)^{2}}
$$

The smaller the magnitude of above estimation, the more likely the pixel will be on the boundary. This is a probability density function with unilateral Cauchy distribution, and the corresponding random variable is the module of the estimation of boundary.

\section{STUDIES FOR SOME IMPROVED P-M MODELS}

Diffusion filters of P-M model can be divided into two categories, including local gradient based methods [9-12] and local geometrical features methods [13].

For the faults of the recognition of edges in P-M model, the original model can be modified. The diffusion coefficient $\mathrm{g}(\mathrm{s})$ tends to 0 but does not reach 0 , and diffusion coefficients in Eqs5) and (6) are ill-conditioned. This means that although the gradients are large on the edges of images, they are influenced by the pixels in its neighbor, because $\mathrm{g}(\mathrm{s})$ is not equal to zero. And the edges are blurred or destroyed after several iterations even when the influence is small.

Chen [6] introduced Th as a threshold, and let $g(s)=0$ if $\mathrm{s}>\mathrm{Th}$. The modified diffusion coefficient is:

$$
\begin{aligned}
& g^{\prime}(s)=g(s),|s| \leq T h \\
& g^{\prime}(s)=0, \text { others }
\end{aligned}
$$

This new diffusion coefficient can stop diffusing on the edges of images which avoids the blurring of boundaries after several iterations. The improved algorithm is named as "improved thresholding method" in the experiments.

For the computation of difference quotient in P-M model, the control ability of smoothing in diffusion function can be improved. Zhao [7] proposed a rotational coordinate method for the solution of difference quotient. In the original P-M model, the difference quotient can be computed with the top, bottom, left, right and the center pixels in a template sized $3 * 3$, which wastes the other four pixels (top-left, top-bottom, right-left and right-bottom) in the template. Zhao added these four pixels into the computation of the difference quotient (which is equal to a rotation of 45 degrees along the coordinate), and the new discretize formulation of P-M model is:

$$
\begin{aligned}
I_{i, j}^{t+1}=I_{i, j}^{t}+ & \frac{\lambda}{2}\left[g_{N} \bullet \nabla_{N} I+g_{S} \bullet \nabla_{S} I\right. \\
& +g_{E} \bullet \nabla_{E} I+g_{W} \bullet \nabla_{W} I \\
& +g_{1} \bullet \nabla_{1} I+g_{2} \bullet \nabla_{2} I \\
& \left.+g_{3} \bullet \nabla_{3} I+g_{4} \bullet \nabla_{4} I\right]_{i, j}^{t}
\end{aligned}
$$

where, N, S, E, W are the abbreviations for North, South, East, West, respectively; and 1, 2, 3, 4 denote top-left, topbottom, right-left and right-bottom, respectively. In the 
discretized formulation of P-M model, the independent variables of g-function only use four pixels in the $3 * 3$ neighbor template, and the modules of gradients are instead with difference quotients along the four directions, making independent variables contain eight pixels in the neighboring template. With the increase in the neighbor information of the P-M model, the control ability of smoothing is strengthened. The improved algorithm is named as "rotational coordinate method" in the experiments.

The discretized formulation proposed by Perona and Malik is the estimation of the original P-M model, which alters the gradients with the discretized formulation. The modules of the gradients are estimated with the absolutions of the difference quotients within the four-neighbors given in references [3], and [7], the gradient modules $\|\nabla I\|$ are used to obtain the discretization of P-M model:

$$
\begin{gathered}
I_{i, j}^{t+1}=I_{i, j}^{t}+\lambda\left[g_{i, j} \bullet \nabla_{N} I+g_{i, j} \bullet \nabla_{S} I\right. \\
\left.+g_{i, j} \bullet \nabla_{E} I+g_{i, j} \bullet \nabla_{W} I\right]_{i, j}^{t} \\
g_{i, j}=g\left(\left\|\nabla I_{i, j}\right\|\right)
\end{gathered}
$$

This method increases the effect and speed of smoothing. The noisy points can be detected more precisely, and the omission problem of the noisy points in the difference quotient method can be avoided. The improved algorithm is named as "gradient module method" in the experiments.

The designs of diffusion filters can also be modified in the P-M model. The design and selection of the diffusion factor in diffusion equations are very important for implementation. As the estimated functions, the commonly used edge detectors, such as Roberts, Sobel, LoG operators, have similar meanings in statistics.

In a study [8], Gao used two simple horizontal and vertical edges one-ordered differential detectors $\partial \mathrm{I} / \partial x$ and $\partial \mathrm{I} / \partial y$, and the module values of the edge detectors have the same statistical features with the local gradient values: the module values are large on the local edges of the images. Gao constructed the following estimated function:

||$E(x, y, t)||=|\partial u / \partial x|+|\partial u / \partial y|$

and the diffusion function

$g(\|E\|)=1+\cos (\pi \times\|E\| /(\Omega+\|E\|))$

where, $\Omega$ is the threshold of the change rate of diffusion. When the estimated value of edge is smaller than $\Omega$, the change rate of diffusion is large and changes fast; when the estimated value of edge is larger than $\Omega$, the change rate of diffusion is small and tends to zero. Gao's method converges fast [8]. The improved algorithm is named as "gradient statistics method" in the experiments.

In this paper, a novel P-M model is proposed. For the gradient statistics in P-M model diffusion function, the threshold is determined to improve the performance of image smoothing. According to the studies in [6] and [8], the original P-M model has been modified, the convergence rate has been improved, so that the diffusion coefficients stop diffusing on the edges of the images, and the blurring of the boundary is avoided after several iterations.

Firstly, the edges with the threshold are determined, and it is assumed $\mathrm{g}(\mathrm{s})=0$ if $\mathrm{s}>\mathrm{Th}$;

Secondly, motivated by taking the local gradient module values as the edge estimated function directly, which is used in Perona and Malik's method, the diffusion function is modified and the following estimated function is constructed as in $\operatorname{Eq}(10)$.

The improved diffusion function $g^{*}(|| E||)$ in this paper is:

$$
\begin{aligned}
& g^{*}(|| E||)=g(|| E||),|| \partial u / \partial x|+| \partial u / \partial y \| \mid \leq T h \\
& g^{*}(|| E||)=0, \text { others }
\end{aligned}
$$

where

$$
g(|| E||)=1+\cos \left(\frac{\pi \times\|\partial u / \partial x|+| \partial u / \partial y\| \mid}{\Omega+\|\partial u / \partial x|+| \partial u / \partial y\| \mid}\right)
$$

\section{ALGORITHM SIMULATION AND IMAGE DENOISNG EXPERIMENTS}

All the algorithms included in this paper are implemented with VC2010 and Windows7 platform. The core algorithms are implemented with $\mathrm{C}$ programing language, and the loading and smoothing of color BM images are supported. The interface of the software is shown in Fig. (1), and the parameters are selected and the experimental results are shown.

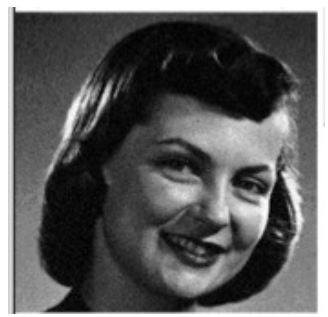

Fig. (1). Software Interface of Image Denoising for Improved P-M model.

In Fig. (2), it can be found that the original image has been contaminated by noises, and the smoothed image has been obtained with P-M model. But the details in the image have been eliminated with the original P-M model, and in the process of denoising, the context in the image has been destroyed. In the following experiments with improved P-M models, the parameters are tuned carefully in order to obtain the best effect of denoising. Experimental results show that the details of the image are maintained well in some experiments, especially in No.3, No.6 and No.9, the details regarding hair have been rarely destroyed. With the improved P-M model, the noisy points are eliminated, and the distortion of the image is small. Compared with experiments No.7 and No.8, the gradient statistic method can eliminate the noisy points, but can blur the image, influencing the quality of the image subjectively. Based on these two experiments, this method embedded threshold 
Table 1. Parameters in experiments.

\begin{tabular}{|c|c|c|c|c|c|c|c|}
\hline 1 & Basic PM & 8 & 20 & 0.25 & Eq. 6 & N/A & N/A \\
\hline 3 & Improved Thresholding & 8 & 20 & 0.12 & Eq.6 & N/A & 18 \\
\hline 4 & Rotational Coordinate & 8 & 20 & 0.12 & Eq.6 & N/A & N/A \\
\hline 6 & Gradient Module & 8 & 20 & 0.12 & Eq. 5 & N/A & N/A \\
\hline 7 & Gradient Statistics & N/A & 15 & 0.09 & Eq.11 & 15 & N/A \\
\hline 8 & Gradient Statistics & N/A & 15 & 0.09 & Eq.11 & 8 & N/A \\
\hline
\end{tabular}

controlling of the image edges into the P-M model, and the results demonstrate that the method improved the quality of the edge and contained more details of the image than the other methods.

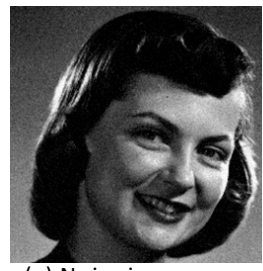

(a) Noisy image

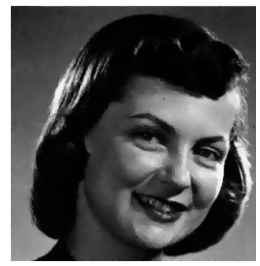

(d) Experment No.3

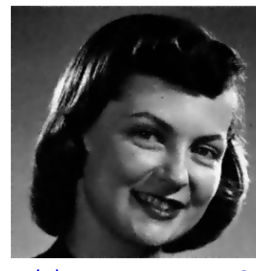

(g) Experment No.6

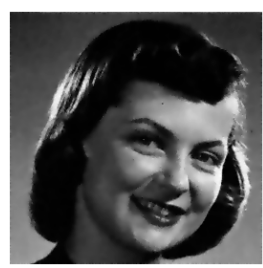

(j) Experment No.9

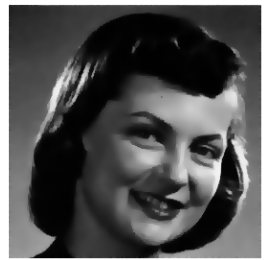

(b) Experment No.1

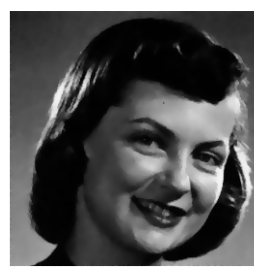

(e) Experment No.4

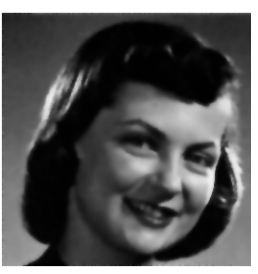

(h) Experment No.7

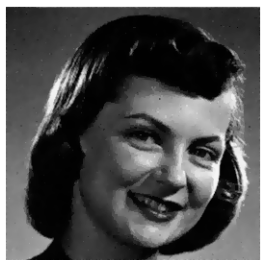

(c) Experment No.2

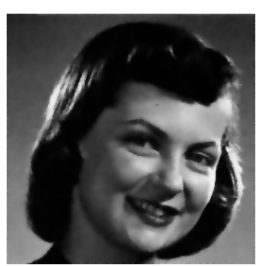

(f) Experment No.5

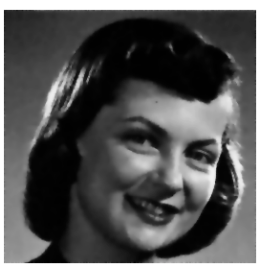

(I) Experment No.8
Fig. (2). The noisy image and the denoised images with PM algorithms in Table $\mathbf{1}$.

Many possible applications can benefit from the modified $\mathrm{P}-\mathrm{M}$ algorithm, including the remote sensing image processing. The image details are well preserved using the P-
$M$ model and the distortion of the image is small. The semantic meaning in the remote sensing images is not destroyed. P-M model can also be used in edge detection, image segmentation and object recognition. All these are important topics in remote sensing image processing.

\section{CONCLUSION}

In this paper, several P-M models were implemented with C-language and the differences were analyzed among these models for image smoothing. A novel P-M model was proposed to determine the gradient statistics in P-M diffusion function with the threshold and improve the smoothing performance of the image.

The P-M model considered the isolated noisy pixels as edge points rather than eliminating them, which rendered the force of smoothing as not enough, as the large modules of the gradients were present at these pixels.

In the procedure of $\mathrm{P}-\mathrm{M}$ diffusion filter, the design and selection of the diffusion-rate function are not uniform and the gradients reflect the edge information of the images. The smaller the magnitude of the image region, the larger the force of the image smoothing, and vice versa. Therefore, the purpose of denoising and remaining edges is achieved, which is the core of the P-M model.

The typical linear edge detector and the edge detectors based on the local geometry features of the images are suitable. The design of diffusion-rate function can be implemented with the technology of fitting statistics distribution. The study of the usage of P-M model in other applications in the image processing and image analysis, such as edge extraction and feature detection, is the added work of this paper.

\section{CONFLICT OF INTEREST}

The authors confirm that this article content has no conflict of interest.

\section{ACKNOWLEDGEMENTS}

Declared none. 


\section{REFERENCES}

[1] A. Goyal, A. Bijalwan, and M.K. Chowdhury, "A comprehensive review of image smoothing techniques", International Journal of Advanced Research in Computer Engineering \& Technology (IJARCET), vol. 1, pp. 315-319, 2012.

[2] L. Xu, C. Lu, Y. Xu, et al. "Image smoothing via L 0 gradient minimization”, ACM Transactions on Graphics (TOG), vol. 30, pp. 174, 2011

[3] L. Pizarro, P. Mrázek, S. Didas, et al. "Generalised nonlocal image smoothing”, International Journal of Computer Vision, vol. 90, pp. 62-87, 2010.

[4] Ö.N. Subakan, and B.C. Vemuri, "A quaternion framework for color image smoothing and segmentation", International Journal of Computer Vision, vol. 91, pp. 233-250, 2011.

[5] P. Perona, and J. Malik, "Scale space and edge detection using anisotropic diffusion", IEEE Transactions on Pattern Analysis and Machine Intelligence, vol. 12, pp. 629-639, 1990.

[6] X. Chen, and Y. P. Lou, "Image smoothing method based on PDE's, computer and modernization”, vol. 5, pp. 17-18, 2004.
[7] F.Q. Zhao, J. Zhi, F. Dai, "Improvement of discrete schme for Perona-Malik model", Journal of Xi'an technological university, vol. 27, pp. 83-87, 2007.

[8] X. Gao, Q. Qin, and R. L. Wang, "Novel anisotropic diffusion filter", Journal of Infrared Millim and Waves, vol. 26, pp. 237-240, 2007.

[9] F. Dai, J. R. Xue, and N. N. Zheng, "Embedding intrinsic mode function into anisotropic diffusion equation for image denoising", Journal of electronics and Information Technology, vol. 30, pp. 509-513, 2008.

[10] M. J. Black, G. Sapiro, D. H. Marimont, and D. Heeger, "Robust anisotropic diffusion", IEEE Transactions on Image Processing, vol. 7, pp. 421-432, 1998.

[11] L. Alvarez, P. L. Lions, and J.M. Morel, "Image selective smoothing and edge detection by nonlinear diffusion", II. SI-AM Journal on Numerical Analysis, vol. 29, pp.845-866, 1992.

[12] F. Catte, P. L. Lions, J. M. Morel, et al. "Image selective smoothing and edge detection by nonlinear diffusion", SI-AM Journal on Numerical Analysis, vol. 29, pp. 182-193, 1992.

[13] A.I. El Fallah, and G. E. Ford, "Mean curvature evolution and surface area scaling in image filtering" IEEE Transactions on Image Processing, vol. 6, pp. 750-753, 1997.

(C) Feng et al.; Licensee Bentham Open.

This is an open access article licensed under the terms of the Creative Commons Attribution Non-Commercial License (http://creativecommons.org/licenses/ by-nc/4.0/) which permits unrestricted, non-commercial use, distribution and reproduction in any medium, provided the work is properly cited. 